\title{
FIXED POINT THEOREMS FOR NONEXPANSIVE MAPPINGS SATISFYING CERTAIN BOUNDARY CONDITIONS ${ }^{1}$
}

\author{
W. A. KIRK
}

ABSTRACT. Let $K$ be a bounded closed convex subset of a Banach space $X$ with int $K \neq \varnothing$, and suppose $K$ has the fixed point property with respect to nonexpansive self-mappings (i.e., mappings $U: K \rightarrow K$ such that $\|U(x)-U(y)\| \leq\|x-y\|, x, y \in K)$. Let $T: K \rightarrow X$ be nonexpansive and satisfy

$$
\text { inf }\{\|x-T(x)\|: x \in \text { boundary } K, T(x) \notin K\}>0 .
$$

It is shown that if in addition, either (i) $T$ satisfies the Leray-Schauder boundary condition: there exists $z \in$ int $K$ such that $T(x)-z \neq \lambda(x-z)$ for all $x \in$ boundary $K, \lambda<1$, or (ii) $\inf \{\|x-T(x)\|: x \in K\}=0$, is satisfied, then $T$ has a fixed point in $K$.

1. Introduction. Let $X$ be a Banach space and $K$ a subset of $X$. In this paper we consider the nonexpansive mappings, a class of mappings $T$ : $K \rightarrow X$ satisfying $\|T(x)-T(y)\| \leq\|x-y\|, x, y \in K$. In 1965 we obtained the following fixed point theorem for this class by invoking the assumption that the domain possess a property Brodskii and Mil 'man [2] call 'normal structure'.

Theorem 1.1 [10]. Let $K$ be a nonempty weakly compact convex subset of the Banach space $X$ and suppose $K$ has normal structure. Then every nonexpansive mapping $T: K \rightarrow K$ has a fixed point.

The assumption of normal structure is a technical one which asserts that every convex subset $A$ of $X$ which has positive diameter must contain a point $x$ such that $\sup \{\|x-y\|: y \in A\}<\operatorname{diam} A$. This assumption, which has been studied in some detail by several authors, always holds for convex sets as well as for bounded convex subsets of uniformly convex spaces

Received by the editors March 18, 1974.

AMS (MOS) subject classifications (1970). Primary $47 \mathrm{H} 10$.

Key words and phrases. Nonexpansive mapping, fixed point theorem, LeraySchauder boundary condition.

${ }^{1}$ Research supported by National Science Foundation grant GP-18045. 
(cf. [2], [13]). It was in this latter setting that F. Browder and D. Göhde, also in 1965, independently obtained the same result as Theorem 1.1 (see [3], [8]).

A number of the subsequent generalizations of Theorem 1.1 have been derived by weakening the assumption that $T: K \rightarrow K$. For example in [11] it is shown that this as sumption may be weakened to $T: K \rightarrow H$, where $H$ is any convex set containing $K$, provided $T$ map the relative boundary of $K$ in $H$ back into $K$. Similar results for the more general 'pseudo-contractive mappings' are found in Assad-Kirk [1] and M. G. Crandall [6]. Even more recently, S. Reich [16] and G. Vidossich [18] have shown (also for the pseudocontractive mappings) that one only need assume in Theorem 1.1 that $T$ : $K \rightarrow X$ be 'inward' in the sense of Halpern and Bergman [9] (i.e., if $x \in K$ and $T(x) \neq x$ then the segment $[x, T(x)]$ contains a point of $K$ distinct from $x)$. In each of the above generalizations the setting may be taken as prescribed in Theorem 1.1 and with the exception of Crandall's result, which uses a common fixed point theorem for a commutative semigroup of transformations of $K \rightarrow K$, the methods of proof amount to a reduction of the problem to an application of Theorem 1.1. Our purpose in this paper is to note that a further such weakening of the assumption $T: K \rightarrow K$ is possible, one which also may be formulated in this same setting.

2. The boundary condition ( $L-S$ ). In contrast to the weakenings described above there is a weakening of the assumption $T: K \rightarrow K$ which appears to require the stronger uniformly convex setting; this is the LeraySchauder boundary condition imposed by Browder in [4] and defined as follows:

The mapping $T: K \rightarrow X$ is said to satisfy (L-S) on $\partial \subset K$ if:

(L-S) There exists $z \in$ int $K$ such that $T(x)-z \neq \mu(x-z)$ for $x \in \partial$ and $\mu>1$.

This assumption has been imposed by several authors recently in proving fixed point theorems for 'condensing' and ' $k$-set contractive' mappings (e.g. [12], [14], [15], [20]). Browder's original result (for nonexpansive mappings) is the following:

Theorem 2.1 [4]. Suppose $K$ is a bounded closed convex subset of a uniformly convex Banach space $X$ with $0 \in$ int $K$, and suppose $T: K \rightarrow X$ is nonexpansive and satisfies $T x \neq \mu x$ for $x$ in the boundary of $K$ and $\mu>1$. Then $T$ has a fixed point in $K$.

The above theorem (stated in [4] for the more general semicontractive 
mappings) is not derived from the earlier theorems for mappings $T: K \rightarrow K$, but rather it is a consequence of the fact that $I-T$ is demiclosed on $K$ (Theorem 3 of [4]). It is in the proof of this latter theorem that Browder makes crucial use of the structure of uniform convexity on $X$.

3. Results. The use of the condition (L-S) in Theorem 2.1 and our partially successful attempt to replace the assumption $T: K \rightarrow K$ in Theorem 1.1 with this assumption motivate this report. In the generalization of Theorem 1.1 we give below (Theorem 3.1) we find it necessary however to impose another condition in addition to the condition (L-S) on the boundary, $\partial K$, of $K$.

Theorem 3.1. Let $K$ be a bounded closed convex subset of a Banach space $X$ with int $K \neq \varnothing$, and suppose $K$ has the fixed point property with respect to nonexpansive self-mappings. Suppose $T: K \rightarrow X$ is nonexpansive and suppose:

(i) $T$ satisfies (L-S) on $\partial K$, and

(ii) inf $\{\|x-T(x)\|: x \in \partial K, T(x) \notin K\}>0$.

Then $T$ has a fixed point in $K$.

The question remains open as to whether this theorem remains true in the absence of this extra assumption (ii), and thus it is not known whether the assumption of uniform convexity on $X$ in Theorem 2.1 can be replaced with the assumptions on $K$ and $X$ of Theorem 1.1. Note, however, that if int $K \neq \varnothing$, Theorem 3.1 does weaken the domain/range assumptions on $T$ in. Theorem 1.1 because both conditions (i) and (ii) of Theorem 3.1 hold if $T: K \rightarrow K$, or even more generally if $T: \partial K \rightarrow K$.

We derive as a direct consequence of Theorem 3.1 the following result.

Theorem 3.2. Let $K$ be a bounded closed convex subset of the Banach space $X$ with int $K \neq \varnothing$, and suppose $K$ has the fixed point property with respect to nonexpansive self-mappings. If $T: K \rightarrow X$ is nonexpansive and satisfies

(*) $\quad \inf \{\|x-T x\|: x \in K\}<\inf \{\|x-T x\|: x \in \partial K, T x \notin K\}$,

then $T$ has a fixed point in $K$.

Proof of Theorem 3.1. By assumption there exists $z \in$ int $K$ such that $T(x)-z \neq \mu(x-z)$ for $x \in \partial=\partial K, \mu>1$, and there is no loss in generality in assuming $z=0$. First we show that together this fact and (ii) imply

(iii) $r=\inf \{\|T(x)-\mu(x)\|: x \in \partial, T(x) \notin K, \mu>1\}>0$. 
For, suppose $r=0$. Then there exist $\left\{x_{n}\right\} \subset \partial$ with $T\left(x_{n}\right) \notin K$, and $\left\{\mu_{n}\right\} \subset$ $(1, \infty)$ such that $\left\|T\left(x_{n}\right)-\mu_{n} x_{n}\right\| \rightarrow 0$ as $n \rightarrow \infty$. However, since $0 \notin \partial$ there exists $\rho>0$ such that $\left\|x_{n}\right\| \geq \rho, n=1,2, \cdots$. Also because $K$ is bounded there exists a number $M$ such that if $x \in K,\|x\| \leq M$; thus

$$
\|T(x)\| \leq\|T(x)-T(0)\|+\|T(0)\| \leq\|x\|+\|T(0)\| \leq M+\|T(0)\| .
$$

Since $\mu_{n}\left\|x_{n}\right\|-\left\|T\left(x_{n}\right)\right\| \rightarrow 0$ and

$$
\left\|T\left(x_{n}\right)\right\| /\left\|x_{n}\right\| \leq(M+\|T(0)\|) / \rho, \quad n=1,2, \cdots,
$$

the sequence $\left\{\mu_{n}\right\}$ is bounded.

Thus (by choosing a subsequence) we may suppose $\left\{\mu_{n}\right\}$ converges, say $\mu_{n} \rightarrow \mu \geq 1$ as $n \rightarrow \infty$. It follows that $\left\|\mu x_{n}-T\left(x_{n}\right)\right\| \rightarrow 0$, and if $\mu=1$ then (ii) is contradicted. So we may suppose $\mu>1$. To show that this also leads to a contradiction, observe that

yielding

$$
\begin{aligned}
\left\|\mu x_{n}-\mu x_{m}\right\| & =\left\|\mu x_{n}-T\left(x_{n}\right)+T\left(x_{n}\right)-T\left(x_{m}\right)+T\left(x_{m}\right)-\mu x_{m}\right\| \\
& \leq\left\|\mu x_{n}-T\left(x_{n}\right)\right\|+\left\|x_{n}-x_{m}\right\|+\left\|T\left(x_{m}\right)-\mu x_{m}\right\|,
\end{aligned}
$$

$$
(\mu-1)\left\|x_{n}-x_{m}\right\| \leq\left\|\mu x_{n}-T\left(x_{n}\right)\right\|+\left\|T\left(x_{m}\right)-\mu x_{m}\right\| \rightarrow 0 \text { as } n, m \rightarrow \infty .
$$

Since $\mu-1>0$ it follows that $\left\{x_{n}\right\}$ is a Cauchy sequence which must converge, say to $x_{0} \in \partial$. Thus $\mu x_{n}-T\left(x_{n}\right) \rightarrow \mu x_{0}-T\left(x_{0}\right)=0$, and this contradicts (i). Therefore (iii) is proved.

To complete the proof, let $\eta=(M+\|T(0)\|) / \rho$ with $M$ and $\rho$ as above, and let $\lambda \in(0,1)$ satisfy

$$
\lambda r-(1-\lambda) M(\eta+1)>0 .
$$

Let $y^{*} \in(1-\lambda) K$ and define $U_{\lambda}: K \rightarrow X$ by $U_{\lambda}(x)=\lambda T(x)+y^{*}$. Suppose $T(x) \notin K$. Then if $U_{\lambda}(x)=\alpha x$ for $x \in \partial$ and $\alpha>1$ we have

$$
\alpha=\left\|\lambda T(x)+y^{*}\right\| /\|x\| \leq(M+\|T(0)\|) / \rho=\eta,
$$

and hence (using (iii))

$$
\begin{aligned}
\left\|U_{\lambda}(x)-\alpha x\right\| & \geq \lambda\|T(x)-\alpha x\|-\|\alpha x-\lambda \alpha x\|-\left\|y^{*}\right\| \\
& \geq \lambda r-(1-\lambda) \alpha\|x\|-\left\|y^{*}\right\| \geq \lambda r-(1-\lambda) \alpha M-(1-\lambda) M \\
& =\lambda r-(1-\lambda) M(\alpha+1) \geq \lambda r-(1-\lambda) M(\eta+1)>0 .
\end{aligned}
$$

Also, if $T(x) \in K$ then, since $y^{*}=(1-\lambda) z$ for some $z \in K$, it follows that $U_{\lambda}(x) \in K$. Thus in either case $U_{\lambda}(x) \neq \alpha x$ if $x \in \partial$ and $\alpha>1$. Since $U_{\lambda}$ : 
$K \rightarrow X$ is a contraction mapping, Theorem 5 of [4] (also see [14]) implies that there exists $x^{*} \in K$ such that $U_{\lambda}\left(x^{*}\right)=x^{*}$; hence $\lambda T\left(x^{*}\right)+y^{*}=x^{*}$ and $(I-\lambda T)\left(x^{*}\right)=$ $y^{*}$, proving $I-\lambda T$ maps $K$ onto $(1-\lambda) K$. But $(I-\lambda T)^{-1}$ exists because nonexpansiveness of $T$ implies

$$
\|(I-\lambda T)(u)-(I-\lambda T)(v)\| \geq\|u-v\|-\lambda\|T(u)-T(v)\|
$$

$$
\geq(1-\lambda)\|u-v\|, u, v \in K
$$

Thus $(I-\lambda T)^{-1}:(1-\lambda) K \rightarrow K$, and it follows that if $H=(1-\lambda)(I-\lambda T)^{-1}$ then

$$
H:(1-\lambda) K \rightarrow(1-\lambda) K,
$$

and moreover (+) shows that $H$ is nonexpansive. Since $K$ has the fixed point property with respect to nonexpansive self-mappings, the mapping $\tilde{H}$ : $K \rightarrow K$ defined by

$$
\tilde{H}(x)=(1-\lambda)^{-1} H((1-\lambda) x), \quad x \in K,
$$

has a nonempty fixed point set in $K$. It follows that $H$ has a nonempty fixed point set $S$ in $(1-\lambda) K$. Thus $\cdot T$ has $(1-\lambda)^{-1} S$ as a fixed point set, for if $H(z)=z$ then

$$
(I-\lambda T)(z /(1-\lambda))=z
$$

yielding

$$
z /(1-\lambda)-\lambda T(z /(1-\lambda))=z,
$$

and hence $T(z /(1-\lambda))=z /(1-\lambda)$.

Theorem 3.2 actually is a direct consequence of Theorem 3.1. To see this observe that $(*)$ implies condition (ii) immediately, and the following shows that it also implies condition (i).

Proposition. Let $K$ be a closed convex subset of a Banach $X$ with int $K \neq \varnothing$. If $T: K \rightarrow X$ is nonexpansive and satisfies (*), then $T$ satisfies (L-S) on $\partial K$.

Proof. Choose $z \in$ int $K$ so that $\|z-T(z)\|<r$ where

$$
r=\inf \{\|x-T(x)\|: x \in \partial K, T(x) \notin K\} .
$$

Now suppose $T(x)-z=\mu(x-z)$ for some $x \in \partial K, \mu>1$. Then

$$
\|T(x)-x\|=\|(1-\mu)(z-x)\|=(\mu-1)\|z-x\| .
$$

But

$$
\|T(x)-z\| \leq\|T(x)-T(z)\|+\|T(z)-z\|<\|x-z\|+r
$$


and $\|T(x)-z\|=\mu\|x-z\|$, so $\mu\|x-z\|<\|x-z\|+r$. Thus

$$
(\mu-1)\|x-z\|<r
$$

and this implies $\|T(x)-x\|<r$. This can happen only if $T(x) \in K$, but $T(x)-z^{\prime}=\mu(x-z), \mu>1$, implies $T(x) \notin K-$ a contradiction.

As a corollary of Theorem 3.1 we have the following known result, first noticed by Browder [5]. Browder's result which is also given in [19], yields more. It actually shows that for $T$ lipschitzian, $p \epsilon$ int $K$, and $\epsilon>0$ sufficiently small, $\epsilon T+(1-\epsilon) p$ maps $K$ into $K$ (if $T: \partial K \rightarrow K$ ).

Corollary. Suppose $K$ is a bounded closed convex subset of the Banach space $X$ and suppose $K$ has the fixed point property with respect to nonexpansive self-mappings. If $T: K \rightarrow X$ is nonexpansive, and if $T: \partial K \rightarrow K$, then $T$ has a fixed point in $K$.

Proof. Because $T(x) \in K$ for $x \in \partial K$, condition (i) of Theorem 3.1 is trivially satisfied and condition (ii) is satisfied vacuously.

We wish to thank R. L. Thele and the referee for suggesting improvements in our assumptions.

\section{REFERENCES}

1. N. A. Assad and W. A. Kirk, Fixed point theorems for set-valued mappings of contractive type, Pacific J. Math. 43 ( 1972 ), 553-562.

2. M. S. Brodskii and D. P. Mil' 'man, On the center of a convex set, Dokl. Akad. Nauk SSSR 59 (1948), 837-840. (Russian) MR 9, 448.

3. F. E. Browder, Nonexpansive nonlinear operators in a Banach space, Proc. Nat. Acad Sci. U.S.A. 54 (1965), 1041-1044. MR 32 \#4574.

4. - Semicontractive and semiaccretive nonlinear mappings in Banach spaces, Bull. Amer. Math. Soc. 74 (1968), 660-665. MR 37 \#5742.

5. R. E. Bruck, Jr., Private communication.

6. M. G. Crandall, Differential equations on convex sets, J. Math. Soc. Japan 22 ( 1970), 443-455. MR 42 \#3388.

7. J. A. Gatica and W. A. Kirk, A fixed point theorem for $k$-set-contractions defined in a cone, Pacific J. Math. 53 (1974), 131-136.

8. D. Göhde, Zum Prinzip der kontraktiven Abbildung, Math. Nachr. 30 (1965), 251-258. MR $32 \# 8129$.

9. B. Halpern and G. Bergman, A fixed point theorem for inward and outward maps, Trans. Amer. Math. Soc. 130 (1968), 353-358. MR 36 \#397.

10. W. A. Kirk, A fixed point theorem for mappings which do not increase distances, Amer. Math. Monthly 72 (1965), 1004-1006. MR 32 \#6436.

11. - Fixed point theorems for nonlinear nonexpansive and generalized contraction mappings, Pacific J. Math. 38 (1971), 89-94. MR 46 \# 4290.

12. R. D. Nussbaum, The fixed point index for local condensing mappings, Ann. Mat. Pura Appl. (4) 89 (197 1), 217-258. MR 47 \#903.

13. Z. Opial, Nonexpansive and monotone mappings in Banach spaces, Lecture Notes 67-1, Brown University, Providence, R. I., 1967.

14. W. V. Petryshyn, Structure of the fixed point sets of $k$-set-contractions, Arch. 
Rational Mech. Anal. 40 (1970/71), 312-328. MR 42 \#8358.

15. - Remarks on condensing and $k$-set-contractive mappings, J. Math. Anal. Appl. 39 ( 1972), $717-741$.

16. S. Reich, Remarks on fixed points, Atti Accad. Naz. Lincei Rend. Cl. Sci. Fis. Mat. Natur. 52 ( 1972), 690-697.

17. - Fixed points of condensing functions, J. Math. Anal. Appl. 41 (1973), 460-467.

18. G. Vidossich, Nonexistence of periodic solutions of differential equations and applications to zeros of nonlinear operators (preprint).

19. - Applications of topology to analysis: On the topological properties of the set of fixed points of nonlinear operators, Confer. Sem. Mat. Univ. Bari 126 (1971), 1-62.

20. J. R. L. Webb, A fixed point theorem and applications to functional equations in Banach spaces, Boll. Un. Mat. Ital. (4) 4 (1971), 775-778.

DEPARTMENT OF MATHEMATICS, UNIVERSITY OF IOWA, IOWA CITY, IOWA 52242 